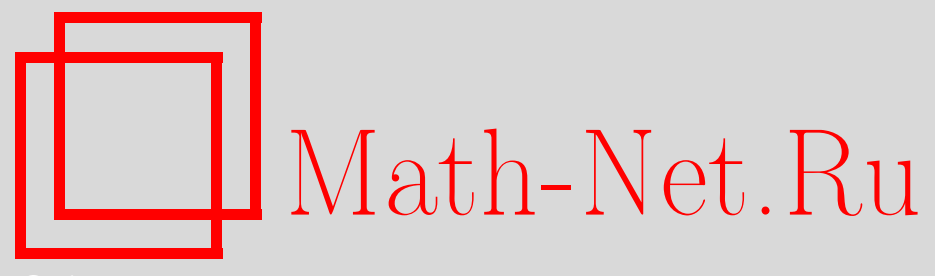

Е. Г. Репина, Регрессионная математическая модель влияния микрофинансовых организаций на интенсивность развития малого и среднего предпринимательства в регионах Российской Федерации, Вестн. Сам. гос. техн. ун-та. Сер. Физ.мат. науки, 2013, выпуск 4(), 131-137

DOI: https://doi.org/10.14498/vsgtu1287

Использование Общероссийского математического портала MathNet.Ru подразумевает, что вы прочитали и согласны с пользовательским соглашением

http://www . mathnet.ru/rus/agreement

Параметры загрузки:

IP : 44.207 .124 .84

26 апреля 2023 г., 13:47:42

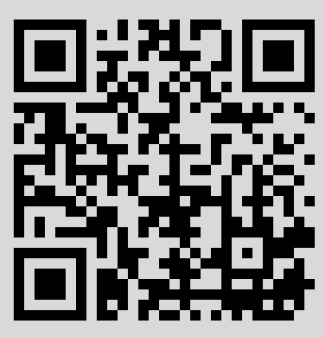




\title{
РЕГРЕССИОННАЯ МАТЕМАТИЧЕСКАЯ МОДЕЛЬ ВЛИЯНИЯ МИКРОФИНАНСОВЫХ ОРГАНИЗАЦИЙ НА ИНТЕНСИВНОСТЬ РАЗВИТИЯ МАЛОГО И СРЕДНЕГО ПРЕДПРИНИМАТЕЛЬСТВА В РЕГИОНАХ РОССИЙСКОЙ ФЕДЕРАЦИИ
}

\author{
E. Г. Репина \\ Самарский государственный экономический университет, \\ 443090, Россия, Самара, ул. Советской Армии, 141. \\ E-mail: violet261181@mail.ru
}

\begin{abstract}
Проведена многомерная статистическая группировка микрофинансовых организаций, зарегистрированных на территории Российской Федераџии, по типу организачионно-правовой бормы хозяйствования. Разработана и исследована регрессионная модель, отражающая влияние числа микробинансовых организащий на интенсивность развития малого и среднего бизнеса в регионах.
\end{abstract}

Ключевые слова: регрессионная модель, микрофинансовая организачия, организационно-правовая форма.

Роль такого субъекта экономического пространства, как микрофинансовая организация (МФО), на современном этапе развития экономики трудно переоценить. Особенности развития микрофинансовых организаций, тенденции изменения их количества по субъектам Российской Федерации отражены в работе [1].

Целью настоящего исследования является статистический анализ структуры МФО по организационно-правовой форме, а также изучение влияния количества МФО, зарегистрированных на территории субъекта РФ, на определённые региональные экономические показатели.

Базой исследования явился статистический массив, сформированный на основе официальных статистических данных, собранных по субъектам РФ [2], а также отдельно по Самарской области [3].

Анализ разновидностей организационно-правовых форм МФО проводился на основе данных табл. 1, которая является результатом многомерной типологической группировки по организационно-правовой форме и принадлежности организации конкретному федеральному округу. Для группировки использовались данные государственного реестра микрофинансовых организаций по состоянию на 20.12.2012, опубликованные на официальном сайте федеральной службы по финансовым рынкам РФ (http://www.fcsm.ru/ $\mathrm{ru} /$ contributors/microf inance_org/state_register_SRO_microfinance_org/).

Анализ данных табл. 1 показал, что максимальное число МФО зарегистрировано на территории Приволжского федерального округа (28,8 \%), минимальное - в Северо-Кавказском федеральном округе (3,33\%). Наиболее распространённой организационно-правовой формой является «Общество с ограниченной ответственностью» - 87,07\% всех зарегистрированных в РФ микрофинансовых организаций. Второй по численности формой является «Фонд поддержки предпринимательства»-6,43\%, причём такие фонды мо-

Евгения Геннадъевна Репина (к.э.н., доц.), доцент, каф. математической статистики и эконометрики. 


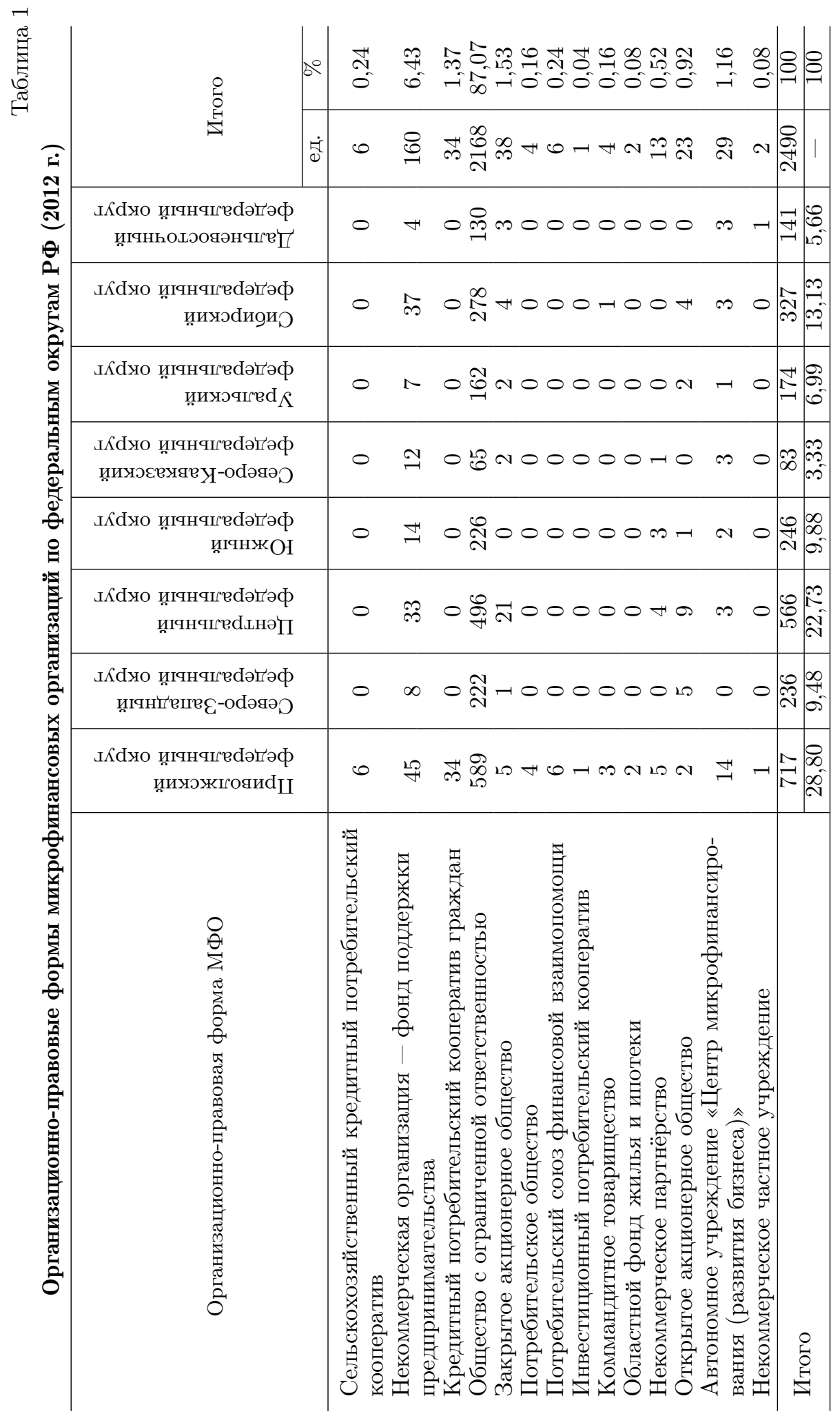


гут быть как областными, так и относящимися к конкретному городскому образованию. Более 1 \% всех зарегистрированных МФО составляют «Кредитный потребительский кооператив граждан» (1,37\% организаций зарегистрированы только в Приволжском федеральном округе), «Закрытое акционерное общество» $(1,53 \%)$, автономное учреждение «Центр микрофинансирования (развития бизнеса)» $(1,16 \%)$. Прочие организационно-правовые формы, приведённые в табл. 1, наименее распространены (их суммарная доля - 2,44\%). На территории РФ зарегистрирован всего 1 инвестиционный потребительский кооператив.

На уровень развития экономики той или иной территории, безусловно, влияет число зарегистрированных малых и средних предприятий (включая индивидуальных предпринимателей). Развитие малого и среднего бизнеса также ведёт к эффективному ресурсосберегающему экономическому росту [4].

На базе данных государственной статистики $[2,3]$ был собран массив статистических данных по 83 субъектам РФ относительно таких показателей, как «Число микрофинансовых организаций» $(X$, ед.) и «Число субъектов малого и среднего предпринимательства (юридические лица и индивидуальные предприниматели)» ( $Y$, ед.). Такой субъект федерации, как г. Москва, был исключён из исследования в силу его «нетипичности»- резкого отличия от других элементов статистического массива. В качестве обоснования приведённого утверждения была применена непараметрическая статистика типа Граббса [5]. По показателю «Число микрофинансовых организаций» г. Москва отличается значением, равным 293. Исходя из нормальности распределения данного показателя было рассчитано наблюдаемое значение по формуле [5]

$$
G_{\text {набл }}=\frac{X_{\max }-\bar{X}}{\sqrt{\frac{1}{n-1} \sum_{i=1}^{n}\left(X_{i}-\bar{X}\right)^{2}}} .
$$

Соответственно, $G_{\text {набл }}=6,73, G_{\text {кр }}(\alpha=0,01 ; n=83)=3,17$. При попада-

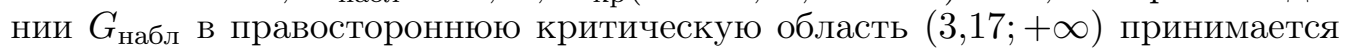
альтернативная гипотеза о существенном отличии рассматриваемого «нетипичного» значения от прочих элементов исследуемой совокупности.

Графический анализ дискретной информации зависимости $Y$ от $X$ по 83 регионам (рис. 1) позволил предположить наличие прямой линейной зависимости между числом МФО $(X$, ед.) в определённом федеральном округе и количеством зарегистрированных на его территории малых и средних предприятий $\left(Y\right.$, ед.). Линейный коэффициент корреляции $r_{\text {в }}=0,79$, что свидетельствует о тесной взаимосвязи показателей. Проверка статистической значимости коэффициента с использованием случайной величины $t$, имеющей распределение Стьюдента с числом степеней свободы $k=80$, дала положительный результат $\left(t_{\text {набл }}=11,44 ; t_{\mathrm{Kp}}(\alpha=0,05 ; k=80)=1,99\right)$.

Начальным этапом эконометрического (статистического) анализа является построение линейного уравнения парной регрессии вида

$$
y=b_{0}+b_{1} x+\varepsilon
$$

где $\varepsilon$ - случайная величина. Оценки теоретических коэффициентов регрессии получены по методу наименьших квадратов, и выборочное регрессионное 


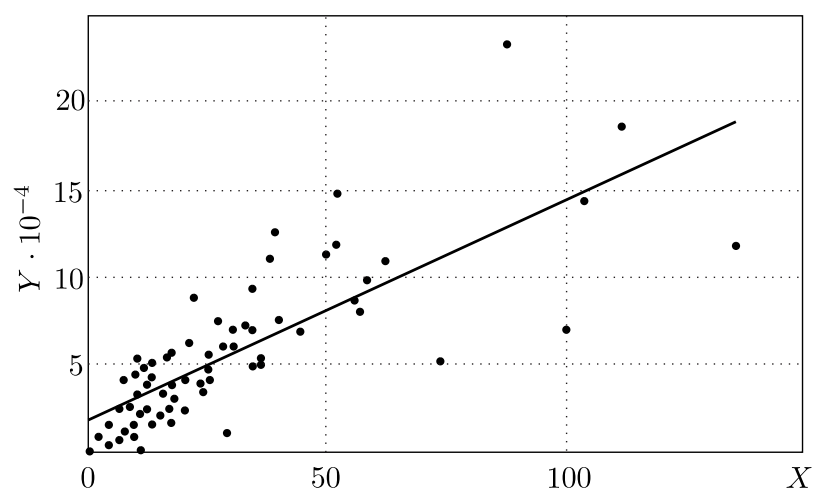

Рис. 1. Корреляционное поле зависимости «Число микрофинансовых организаций» $(X$, ед.) - «Число субъектов малого и среднего предпринимательства» $(Y$, ед.)

уравнение, представленное на рис. 1 прямой линией, имеет вид

$$
\hat{y}=18005,41+1264,14 x .
$$

При построении модели высказано предположение о влиянии числа МФО на численность предприятий малого и среднего бизнеса, что даёт основание выдвинуть нулевую гипотезу $H_{0}: b=0$ об отсутствии такового. Рассчитанное значение статистики Стьюдента $\left(t_{n}=\hat{b}_{1} / S_{\hat{b}_{1}}=11,43 ; t_{\mathrm{kp}}(\alpha=0,05 ; k=80)=\right.$ $=1,99)$ позволило опровергнуть гипотезу $H_{0}$. Таким образом, оценка $\hat{b}_{1}=$ $=1264,14$ статистически значима. Свободный член уравнения $(1) \hat{b}_{0}=18005,41$ также был признан статистически значимым $\left(t_{n}=\hat{b}_{0} / S_{\hat{b}_{0}}=4,32 ; t_{\mathrm{\kappa p}}(\alpha=0,05\right.$; $k=80)=1,99)$.

После построения регрессионного уравнения были более детально рассмотрены остатки в каждом наблюдении $\left(e_{i}=y_{i}-\hat{y}_{i}\right)$. Вычислен коэффициент детерминации $R^{2}=0,62 . F$-критерий позволил считать его значимым $\left(F_{\text {набл }}=130,8 ; F_{\mathrm{кp}}\left(\alpha=0,05 ; k_{1}=1 ; k_{2}=80\right)=3,96\right)$. Следовательно, построенная статистическая модель (1) удовлетворительно аппроксимирует результаты наблюдений.

Статистическая эффективность полученных МНК-оценок регрессионного уравнения (1), т. е. отсутствие гетероскедастичности (выполнение условия $\left.D\left(\varepsilon_{i}\right)=D\left(\varepsilon_{j}\right)=\sigma_{\varepsilon}^{2}, i \neq j\right)$, в процессе моделирования проверялась на основе анализа дискретной графической зависимости точек $\left(x_{i}, e_{i}^{2}\right)$ (рис. 2).

Анализ расположения точек $\left(x_{i}, e_{i}^{2}\right)$ на рис. 2 позволяет выдвинуть гипотезу о наличии положительной гетероскедастичности вида

$$
\sigma_{\varepsilon}=\gamma+\beta x^{\vartheta}
$$

На 5-процентном уровне значимости критерия Глейзера [6,7] позволил установить конкретную взаимосвязь колебаний случайной компоненты $\sigma_{\varepsilon}$ в зависимости от величины факторного признака $x^{\vartheta}$. Результаты численного вариативного исследования приведены в табл. 2 .

Наиболее значимым по результатам применения $t$-статистики $\left(t_{\mathrm{\kappa p}}(\alpha=0,05\right.$; $k=80)=1,99)$ из возможных оценок $\hat{\beta}$ является значение, соответствующее 


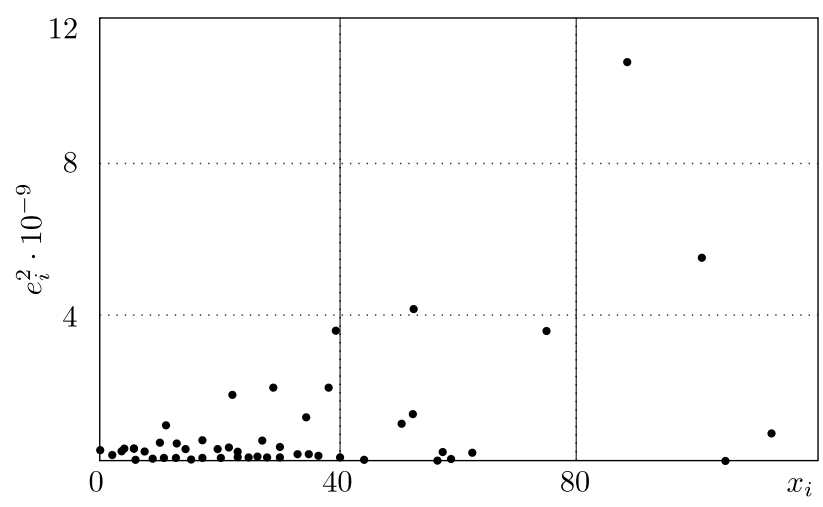

Рис. 2. Дискретная графическая зависимость $\left(x_{i}, e_{i}^{2}\right)$

Таблица 2

Результаты оценивания зависимости $\sigma_{\varepsilon}=\gamma+\boldsymbol{\beta} \boldsymbol{x}^{\vartheta}$ для различных $\boldsymbol{\vartheta}$

\begin{tabular}{c|c|c|c|c|c|c}
\hline$\vartheta$ & $\hat{\beta}$ & $\gamma$ & $S_{\hat{\beta}}$ & $S_{\gamma}$ & $R^{2}$ & $t_{\text {набл }}$ \\
\hline-2 & $-67762,6$ & 18377,91 & 71729,68 & 2184,818 & 0,0111 & $-0,94469$ \\
$-1,8$ & $-68226,4$ & 18648,95 & 61216,51 & 2228,004 & 0,01548 & $-1,11451$ \\
$-1,6$ & $-69081,1$ & 19077,6 & 51863,94 & 2297,681 & 0,021964 & $-1,33197$ \\
$-1,3$ & $-70133,4$ & 20242,82 & 39727,1 & 2492,326 & 0,037953 & $-1,76538$ \\
$-0,8$ & $-68078,6$ & 25429,77 & 24386,86 & 3400,856 & 0,089789 & $-2,79161$ \\
$-0,5$ & $-67089,7$ & 34095,7 & 18961,29 & 5014,464 & 0,136793 & $-3,53825$ \\
$-0,2$ & $-88993,4$ & 67361,44 & 20698,31 & 11694,41 & 0,189628 & $-4,29955$ \\
0,5 & 4829,542 & $-5645,33$ & 844,8899 & 4450,981 & 0,292588 & 5,716179 \\
0,8 & 1069,279 & 3268,695 & 177,0865 & 2953,11 & 0,315778 & 6,038177 \\
0,9 & 659,4612 & 4998,024 & 108,074 & 2705,236 & 0,320335 & 6,101939 \\
$\mathbf{1 , 2}$ & $\mathbf{1 5 7 , 1 0 9}$ & $\mathbf{8 5 5 5 , 6 2}$ & $\mathbf{2 5 , 4 4 0 9}$ & $\mathbf{2 2 6 8 , 9 3}$ & $\mathbf{0 , 3 2 5 5 7}$ & $\mathbf{6 , 1 7 5 4 4}$ \\
1,23 & 136,1626 & 8820,586 & 22,05343 & 2241,328 & 0,325483 & 6,174214 \\
1,25 & 123,7729 & 8990,397 & 20,05185 & 2224,052 & 0,325372 & 6,172642 \\
1,3 & 97,50319 & 9392,658 & 15,81258 & 2184,459 & 0,324912 & 6,166177 \\
1,5 & 37,48799 & 10735,33 & 6,136879 & 2066,977 & 0,320812 & 6,10864 \\
1,8 & 8,874303 & 12173,8 & 1,490647 & 1969,898 & 0,30969 & 5,953324 \\
\hline
\end{tabular}

степени $\vartheta=1,2$ (соответствующая строка в табл. 2 выделена). Следовательно, в статистической модели (1) нарушается второе условие Гаусса-Маркова, так как присутствует гетероскедастичность вида

$$
\sigma_{\varepsilon}=8555,62+157,109 x^{1,2} .
$$

Для устранения проблемы гетероскедастичности остатков в процессе исследования применён обобщённый метод наименьших квадратов с использованием критерия Глейзера [7, стр. 688]. В результате оценивания модели вида

$$
\frac{y}{\sigma_{\varepsilon}}=b_{0} \frac{1}{\sigma_{\varepsilon}}+b_{1} \frac{x}{\sigma_{\varepsilon}}+\frac{\varepsilon}{\sigma_{\varepsilon}}
$$

получены следующие уточнённые оценки теоретических коэффициентов $b_{0}$ и $b_{1}: \hat{b}_{0}=11279,2 ; \hat{b}_{1}=1567,30$. Выборочное уравнение регрессии принимает вид

$$
\tilde{y}_{l}=11279,2+1567,30 \tilde{x}_{l} .
$$


Приведённые оценки статистически значимы, коэффициент детерминации достигает $85,65 \%\left(R^{2}=0,8565\right)$. Повторная проверка на выполнение второго условия Гаусса-Маркова даёт положительный результат.

Кроме этого, в процессе моделирования решена проблема незначительной автокорреляции ошибок регрессии. Оценка теоретического коэффициента автокорреляции первого порядка составила $\hat{p}=0,26$. Гипотеза о наличии автокорреляции $(\alpha=0,05)$ принята на основе расчёта статистики ДарбинаУотсона $(D W)$ [8, стр. 219-221]. Наблюдаемое значение статистики $D W=$ $=1,46 ; d_{1}=1,61 ; d_{2}=1,67$.

В качестве инструмента для решения проблемы автокорреляции применяется авторегрессионная схема первого порядка $\varepsilon_{i}=\hat{p} \varepsilon_{i-1}+\vartheta_{i}$ с процедурой восстановления первого наблюдения с помощью поправки Прайса-Уинстона $\sqrt{1-\hat{p}^{2}}$ [8, стр. 222-223].

В результате были уточнены оценки $\hat{b}_{0}$ и $\hat{b}_{1}$ и окончательный вид регрессионной модели, для которой выполняются все предпосылки MHK, записывается как

$$
\breve{y}=10157,88+1616,17 \breve{x} .
$$

Из модели (2) следует, что увеличение числа МФО в регионе способствует росту числа субъектов малого и среднего бизнеса, в частности, увеличение МФО на 1 единицу приводит к росту количества организаций на 1616 единиц.

Таким образом, в настоящей работе на основании математической модели проанализирована структура микрофинансового сектора РФ относительно организационно-правовой формы функционирующих организаций данной отрасли экономики. Проведено эконометрическое моделирование влияния количества МФО, работающих в регионах, на интенсивность развития сферы малого и среднего предпринимательств.

\section{БИБЛИОГРАФИЧЕСКИЙ СПИСОК}

1. Е. Г. Репина, "Статистическое исследование деятельности микрофинансовых организаций с применением методов эконометрического моделирования" // Вестн. СГЭУ, 2013. № 3(101). C. 98-103. [E. G. Repina, "An statistical study of microfinance institutions using the methods of econometric modeling" // Vestn. SGEU, 2013. no. 3(101). Pp. 98-103].

2. Регионы России. Социально-экономические показатели. М.: Росстат, 2012. 990 c., http://www.gks.ru/wps/wcm/connect/rosstat_main/rosstat/ru/statistics/ publications/catalog/doc_1138623506156. [ Regions of Russia. Social and Economic Indicators. Moscow: Rosstat, 2012. 990 pp.]

3. Самарский статистический ежегодник. Самара: Самарастат, 2013. 358 с., http://samarastat.gks.ru/wps/wcm/connect/rosstat_ts/samarastat/ru/publications/ official_publications/electronic_versions/. [ Samara Statistical Yearbook. Samara: Samarastat, 2013. 358 pp.]

4. И. О. Князев, Роль малого бизнеса в экономике России, http://www.ieay.ru/nauch/ sc_article/2007/01/Knayzev.shtml. [I. O. Knyazev, Role of small business in the Russian economy].

5. Е. В. Зарова, О. А. Репин, Е. Г. Репина, Особенности применения критерия Граббса при проверке выборочных данных на аномальность в экономических исследованиях. Самаpa: СГЭУ, 2006. 92 c. [E. V. Zarova, O. A. Repin, E. G. Repina, Features of the application of of Grubbs criteria for verification of sample data on the abnormality in economic research. Samara: SGEU, 2006. 92 pp.]

6. H. Glejser, "A New Test for Heteroskedasticity" // J. Am. Stat. Assoc., 1969. Vol. 64, no. 325. Pp. 316-323. 
7. С. А. Айвазян, В. С. Мхитарян, Прикладная статистика и основы эконометрики. М.: Юнити-Дана, 2000. 1006 с. [S. A. Aivazian, V. S. Mkhitaryan, Applied statistics and essentials of econometrics. Moscow: Yuniti-Dana, 2000. 1006 pp.]

8. К. Доугерти, Введение в эконометрику. М.: Инфра-М, 1999. xiv+402 с. [C. Dougherty, Introduction to Econometrics. Moscow: Infra-M, 1999. xiv+402 pp.]

Поступила в редакцию 26/VIII/2013;

в окончательном варианте - 25/X/2013.

MSC: 91G70

\section{REGRESSION MATHEMATICAL MODEL OF MICROFINANCE INSTITUTIONS INFLUENCE ON SMALL AND MEDIUM ENTERPRISES DEVELOPMENT INTENSITY IN RUSSIAN FEDERATION REGIONS}

\section{E. G. Repina}

Samara State Economic University,

141, Sovetskoy Armii st., Samara, 443090, Russia.

E-mail: violet261181@mail.ru

The multidimensional statistical grouping of microfinance institutions registered in the territory of the Russian Federation by the type of organizational and legal forms of business is made. The regression econometric model that reflects the influence of the number of microfinance institutions on the intensity of development of small and medium-sized businesses in the regions is developed and investigated.

Keywords: regression model, microfinance institution, organizational and legal form.

Original article submitted 26/VIII/2013; revision submitted $25 / \mathrm{X} / 2013$.

Eugeniya G. Repina (Ph. D. Econom.), Associate Professor, Dept. of Mathematical Statistics \& Econometrics. 